\title{
Análisis exploratorio de las exportaciones de aceites esenciales en Brasil: evidencia desde 2000 hasta 2015
}

\author{
Exploratory analysis of essential oils exports in Brazil: evidence from 2000 to 2015 \\ Lury Yibel Forero-Peñuela ${ }^{*}$, Zina Loren Forero-Peñuela ${ }^{2}$, Cícero Deschamps ${ }^{1}$, \\ Alexandre Alves-Porsse ${ }^{3}$
}

\section{RESUMEN}

En la actualidad existe poca información respecto a los valores de mercado vinculados a las exportaciones de aceites esenciales en el Brasil, por lo que se plantea como objetivo consolidar la información de las exportaciones de aceites esenciales durante los años 2000 hasta 2015. De dicha información se analizó el valor de las exportaciones de 16 aceites esenciales en el Sistema de Análisis de Información sobre Comercio Exterior (AliceWeb), excluyendo aquellos comercializados a gran escala por el país. Para tal fin, se observaron las exportaciones en dólares y kilogramos por cada estado brasileño y fueron examinados los diez primeros aceites esenciales con los valores más altos de exportación en dólares. El análisis de la información arroja que los estados brasileños más representativos fueron San Pablo y Amazonas, donde San Pablo se posiciona como el primer exportador de los 16 productos seleccionados y Amazonas como el segundo en la exportación de palo rosa y reexportación de lavanda. En específico, los aceites esenciales con el mayor valor acumulado de exportaciones en dólares fueron palo rosa, lavanda y vetiver. Finalmente, se concluyó que la heterogeneidad del comportamiento en las exportaciones de los 16 aceites esenciales impidió el establecimiento de un patrón de comportamiento homogéneo para cada uno de ellos.

Palabras clave: plantas aromáticas, producción, comercialización, AliceWeb.

\section{ABSTRACT}

Actually, there is information shortage regarding of trade values in Brazilian essential oils exports, then it aim to consolidate information about essential oils exports during the years 2000 through 2015. It were analysed export values of 16 essential oils items from Data Analysis System of Foreign Trade Information (AliceWeb) database, and their essential oils trade information during 2000 through 2015, nevertheless were avoided essential oils commonly marketed on a large scale on Brazil. They were analysed dollars values exports for each essential oil and essential oil kilograms marketed by each one Brazilian state. It were choose ten essential oils that exhibited higher value exports. São Paulo stands out on export of all 16 products selected with higher kilogram values and Amazonas prominent rosewood export and lavender re-exportation. Specifically, rosewood, lavender and vetiver were higher dollar value exportation between select items. Finally, it was concluded that heterogeneity at sales performance exports from essential oils impedes establish a homogeneous pattern of behaviour in each essential oil.

Key words: aromatic plants, production, marketing, AliceWeb.

\section{Introducción}

Para las plantas aromáticas como ocurre con muchos productos considerados non-commodity, existe una falta de información detallada, como estadísticas sobre el volumen de producción y consumo, valores en las importaciones y exportaciones (Bandoni, 2000). Los datos son siempre estimaciones y ponderaciones sobre la base de la información esporádica y parcial.

1 Universidade Federal do Paraná. Programa de Pos graduación en Agronomía - Producción Vegetal. Curitiba, Paraná, Brasil.

2 Universidade Estadual de Campinas. Programa de Pos graduación en Desarrollo Económico, Espacio y Medio Ambiente. Ciudad Universitaria. Campinas, São Paulo, Brasil.

3 Universidade Federal do Paraná. Programa de Pos graduación en Desarrollo Económico. Curitiba, Paraná, Brasil.

* Autores para correspondencia: lury.forero@ufpr.br, porsse@ufpr.com 
Por esta razón, se encuentra información muy variada y poco estandarizada. Una de las principales causas de este fenómeno, es que la mayor parte de la producción de especies aromáticas proviene de países en vías de desarrollo, donde la información económica tiende a ser ocasional, incompleta y, en general, no es fiel a la realidad.

En las aduanas muchos de estos productos aparecen agrupados o mezclados, y no se encuentran separados por especie o producto, por lo que dificulta la diferenciación precisa de las cantidades en la que cada aceite esencial está presente (Biocomercio Sostenible, 2003).

Verlet (1992) estima que el 75\% de la producción mundial de aceites esenciales se derivan de especies perennes leñosas, y más de la mitad de esta producción son cítricos. El 25\% restante proviene de especies herbáceas, y hay una pequeña contribución de las especies silvestres, que no llega a más del 1 o $2 \%$.

A nivel mundial, diez aceites esenciales representan el $85 \%$ del mercado: naranja, limón, menta, citronela-de-java, cedro, eucalipto, especies con citral como may chang y limonaria, lavanda, lavandins y pinos. En el caso de Brasil, este se ubica como el cuarto país de mayor exportación de aceites esenciales (Erkekdjian, 2002; Ideaconsultora., 2011) con un notable incremento en los últimos años, centrándose principalmente en el aceite esencial de naranja con el $49 \%$ del total de su producción.

Es factible mencionar que la tendencia del mercado de sabores y aromas naturales ha tenido un valioso aumento de consumo a partir de los años 90, con una creciente demanda de consumidores interesados en adquirir productos naturales, orgánicos, rastreados, ecológicos y sostenibles en el tiempo.

\section{Estructura del mercado}

El comercio internacional de los aceites esenciales es controlado por dos grandes sectores: el primero, conformado por las principales compañías de sabores y fragancias y el segundo por las grandes acopiadoras o brokers de materias primas, establecidas principalmente en Estados Unidos, Europa y Japón (Fretes y Martínez, 2011; Khodjet-El-Khil, 2015).

De acuerdo con Lange (1998) y Bandoni (2000) se pueden identificar cuatro tipos de productores de plantas aromáticas y tres tipos de intermediarios - Brokers.
Entre los cuatro productores de plantas aromáticas se identifican los siguientes:

1. Productores grandes que dirigen un promedio mayor a 80/100 hectáreas de cultivo. El mismo productor exporta, tiene contrato de riesgo con las empresas compradoras o poseen un intermediario - Broker. Normalmente son monocultivos y autosuficientes tecnológica y económicamente. Por lo general disponen de su propia planta de destilación y extracción del aceite esencial.

2. Productores medianos con 10/80 hectáreas de cultivo. Comúnmente tienen varios cultivos y al diversificar se protegen a sí mismos por sus debilidades, ya que tienen una reducida oferta, baja competitividad y disponibilidad de producto esporádico. Algunas veces exportan y trabajan con pedidos anticipados y con contratos de riesgos compartidos.

3. Productores pequeños con menos de 10 hectáreas de cultivo. Dependen necesariamente de un Broker, o están asociados con cooperativas o entidades centralizadoras. Comúnmente, el cultivo de plantas aromáticas es una alternativa a la agricultura tradicional dentro de sus propiedades. Al depender de intermediarios - Brokers, difícilmente tienen grandes éxitos comerciales, y es por esta razón que el objetivo de estos cultivos no es el sustento familiar, por el contrario, son contribuciones temporales o estacionales a los ingresos familiares.

4. Productores secundarios. Los aceites esenciales no son su industria principal, por el contrario, son los recursos secundarios que se obtienen para la producción de otro producto, como frutas cítricas, madera, industria del papel y/o follaje de eucalipto. Ellos pueden considerarse los proveedores de materia prima para los intermediarios de las agroindustrias destiladoras.

Posteriormente, la etapa de comercialización se lleva a efecto por medio de agentes locales que normalmente comercializan algunos aceites esenciales al por mayor, o mediante grandes centros de comercialización internacional, que distribuyen grandes cantidades de aceites esenciales.

Existen grandes empresas que usan directamente una determinada producción para el uso exclusivo, o para competir con otros intermediarios-Brokers. 
Respecto a los intermediarios, de acuerdo con Bandoni (2000), Lange (1998) y Weiss (1997), existen tres clases (Brokers, Middlemen o Wholesalers):

1. Brokers que al pedido del cliente, son los que mueven grandes cantidades de materia prima, y esto les permite regular el precio de los aceites esenciales.

2. Grandes empresas u organizaciones transnacionales que actúan como opciones a largo plazo y por ello, ofrecen precios más bajos a los del mercado por el riesgo que comprende la producción a largo plazo.

3. Brokers que compran por ofertas de lotes de mercancía específicas y de forma esporádica, que como consecuencia son pedidos determinados.

UNCTAD (2005), presenta de forma resumida un diagrama de los movimientos y flujos en el mercado de aceites esenciales (Figura 1):

\section{En el mercado de aceites esenciales}

El mercado de aceites esenciales presenta una disparidad con los productos sintéticos o productos similares, puesto que estos determinan el umbral de calidad, cantidad y precio de los aceites esenciales, así no posean las mismas propiedades terapéuticas de los productos naturales (Laszlo, s.f.). Por tanto, se dificulta la venta de un aceite esencial si este puede ser sustituido por un producto sintético de igual o menor valor económico y además con calidad organoléptica similar (Verlet, 1992).
La demanda histórica de los aceites esenciales (AE) ha sido estable (a excepción de algunos esporádicos), especialmente en la industria de AE tradicionales que sigue la expansión de los mercados de productos naturales, el que posee un crecimiento lento pero al parecer sin límite. Las excepciones están vinculadas con aquellos que consiguen un sustituto sintético o nuevos AE que conservan alguna ventaja comparativa. Por ejemplo, el aceite esencial de lavanda costaba de 10 a 12 USD kg $^{-1}$ en 1975 y el valor no ha variado significativamente hasta hoy, ya que se adquiere

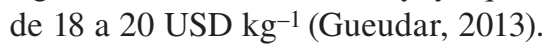

En algunos casos, la estabilidad del mercado es notoria, como el mercado del aceite esencial de geranio, que desde los años 1960 se viene consumiendo en aproximadamente 300 ton año-1 (Erkekdjian, 2002). No obstante, esta misma demanda ha sido abastecida por diferentes países durante el pasar de los años, lo que demuestra un incremento notable en la competencia por calidad, bajos costos de producción y optimización de procesos (SADC, 2005).

Se afirma que la permanencia en el mercado está dada por características como la calidad, cantidad y continuidad. En relación con la calidad, existe un papel primordial en el uso de las normas tanto del productor como del comprador, así como normas públicas nacionales o reglamentos internacionales. Sumado a esto, las variaciones en los precios internacionales son influenciados por factores políticos y climáticos que modifican de forma sustancial la oferta global, principalmente cuando el aceite esencial se produce en una

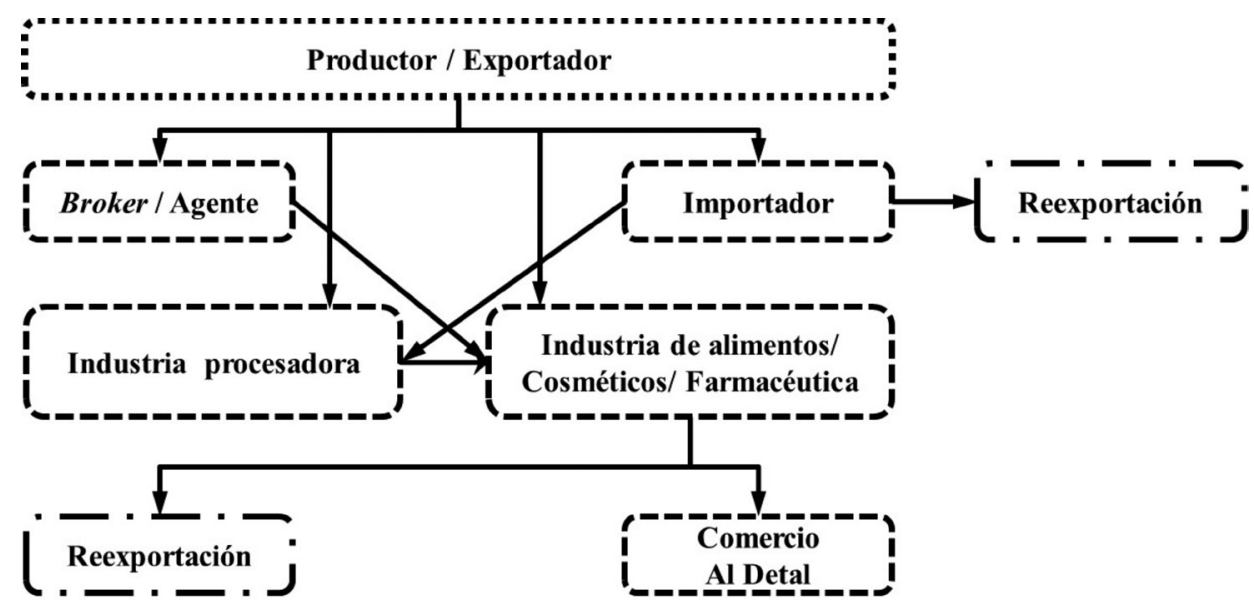

Figura 1. Diagrama de flujo de la comercialización de aceites esenciales (Fuente: UNCTAD, 2005). 
región específica o en pocos países del mundo (Figueiredo, 2008).

Por otro lado, el mercado de aceites esenciales puede ser considerado conservador, ya que tiene cierta desconfianza frente a nuevos productos o calidades, pues difícilmente un comprador acepta algún cambio en la calidad (SADC, 2005), dentro de los patrones que un producto natural puede ofrecer. Sin embargo, paradójicamente, existe una necesidad por la identificación de nuevos productos, como herramienta para innovar y ganar nuevos mercados. En comparación con productos Commodities, los AE tienen elevados precios de venta, con bajos volúmenes de demanda y se encuentran en mercados restringidos. Aun así, existe un alto potencial de usar la diversidad genética y edafoclimática, lo que permitiría hacer de cada región y cada variedad única para la industria de sabores y fragancias (SADC, 2005).

La obtención de productos oriundos de extractivismo, como resinas, concretos, aceites esenciales y absolutos, puede no ser la mejor posibilidad para el desarrollo de una región; sin embargo, se presenta como una alternativa que mayor valor agregado aporta a una planta aromática, estimulando un mayor interés en los sectores económicos latinoamericanos (Bandoni, 2000).

De acuerdo con lo anterior, este artículo tiene como objetivo consolidar informaciones de las exportaciones de aceites esenciales (AE) proporcionadas por AliceWeb durante los años 2000 hasta 2015, con el fin de comprender el mercado de aceites esenciales en Brasil, dejando de lado las especies comúnmente comercializadas a gran escala.

\section{Materiales y Métodos}

Se utilizó la base de datos de comercio desagregada al nivel de ocho dígitos del Sistema Armonizado de Designación y Codificación de Mercancías. Estos datos provienen del Sistema de Análisis de Informaciones de Comercio Exterior, denominado AliceWeb (2016), de la Secretaría de Comercio Exterior, del Ministerio de Desarrollo, Industria y Comercio Exterior brasileño (MDIC). AliceWeb tiene como base de datos el Sistema Integrado de Comercio Exterior, que administra el comercio exterior brasileño y es de libre acceso.

AliceWeb tiene como objetivo principal, auxiliar en la selección de mercados y productos que presentan un mayor potencial para incrementar las exportaciones brasileñas. La información puede encontrarse desagregada ya sea para un determinado estado brasileño, un determinado país de destino de exportación o importación, o información general de todo el país. Las búsquedas pueden ser conjugadas -a criterio del usuario-, dentro de un conjunto de opciones disponibles (Silva et al., 2012).

Al ingresar con el código HS (NCM) -de ocho dígitos- o una palabra clave que identifica a un producto, el sistema proporciona diversas informaciones acerca de este, como el precio promedio, importador potencial, dinamismo, performance de exportación, valores exportados e importados, principales países competidores, medidas arancelarias y no arancelarias.

Se enfatizó en el período de 2000 hasta 2015 , donde se seleccionaron 16 productos pertenecientes a los aceites esenciales (Tabla 1), indicando el número de NCM-SH (Nomenclatura común del MERCOSUR - Sistema Armonizado) y se discriminó las estadísticas sobre el comercio de exportación de cada producto. De este modo, fue segregada la información de los valores acumulados en las exportaciones en dólares para los estados brasileños involucrados, y así mismo los valores acumulados en kilogramos de cada producto de aceite esencial exportado durante cada año.

Se excluyeron los aceites esenciales que se deducen son producidos en forma secundaria por industrias específicas, como la industria de los cítricos (limón, naranja, mandarina, bergamota, petit grain), o la industria del papel que, por lo general, cultivan Pinus spp., Eucalyptus citriodora o Corymbra sp. Igualmente, el código NCM 33012990 llamado "Otros aceites esenciales", fue excluido ya que no era posible discernir cuáles especies estaban involucradas en esta clasificación.

Los valores totales de las exportaciones durante esos 15 años, se organizaron en kilogramos y dólares para cada uno de los productos exportados, y se distinguieron para cada uno de los estados brasileños. Se destacan las 10 primeras posiciones con mayores exportaciones en dólares y se realizó un análisis de forma individual. Por otro lado, fue calculado el valor del kilogramo para los aceites esenciales para los años 2000, 2010 hasta 2015 (Tabla 2). 
Tabla 1. Lista de especies de plantas aromáticas con su respectiva nomenclatura común del Mercosur (NCM) usado en el sitio web AliceWeb para el análisis de los datos de las exportaciones desde 2000 hasta 2015.

\begin{tabular}{llll}
\hline \multicolumn{1}{c}{ NCM } & Nombre común & \multicolumn{1}{c}{ Nombre científico } & Parte da planta \\
\hline 33012918 & Incienso & Myrocarpus frondosus Allemão. & Madera \\
33012912 & Cedro & Cedrela spp. & Madera \\
33012911 & Citronela-de-java & Cymbopogon winterianus Jowitt and Cymbopogon nardus (L.) Rendle & Hojas \\
33012917 & Cilantro & Coriandrum sativum L. & Semillas \\
33012100 & Geranio & Pelargonium hortorum L.H. Bailey & Hojas y flor \\
33012400 & Menta piperita & Mentha piperita L. & Hojas \\
33012200 & Jazmín & Jasminum officinale L. & Hojas y flor \\
$33012300 ; 33012921$ & Lavanda & Lavandula spp. & Hojas y flor \\
33012914 & Limonaria & Cymbopogon citratus (DC.) Stapf & Hojas \\
33012510 & Menta japonesa & Mentha arvensis L. & Hojas \\
33012520 & Menta spearmint & Mentha viridis (L.) L. & Hojas \\
33012590 & Otras mentas & No especificado & Hojas \\
33012916 & Palmarosa & Cymbopogon Martini (Roxb.) Will. Watson & Hojas \\
33012915 & Palo rosa & Aniba roseadora Ducke & Madera y hojas \\
33012913 & Palo santo & Bulnesia sarmientoi Lorentz ex Griseb. & Madera \\
$33012600 ; 33012922$ & Vetiver & Vetiveria zizanioides (L.) Nash & Raíz \\
\hline
\end{tabular}

Tabla 2. Valor del kilogramo en dólares de 16 aceites esenciales seleccionados en el año 2000, 2011 hasta 2015.

\begin{tabular}{lcccccc}
\hline \multirow{2}{*}{ Aceite esencial/Año } & 2000 & 2011 & 2012 & 2013 & 2014 & 2015 \\
\cline { 2 - 7 } & \multicolumn{5}{c}{ USD kg-1 $^{-1}$} \\
\hline Incienso & 62,4 & - & - & - & 48,1 & 50,2 \\
Cedro & 9,7 & 24,3 & 13 & 68,3 & 122 & 38,1 \\
Citronela-de-java & 9,5 & 25,4 & 36,9 & 22 & 5,1 & 19,3 \\
Cilantro & - & - & - & - & 139,2 & - \\
Geranio & 33 & 163,7 & 123,3 & - & - & - \\
Menta piperita & - & 97,1 & 52,5 & 184,1 & 139 & 71 \\
Jazmín & - & - & - & - & - & - \\
Lavanda & 144 & 17,1 & - & 37 & 42 & 18 \\
Limonaria & 30,8 & 8,8 & 49,7 & - & - & - \\
Menta japonesa & - & - & - & 57,1 & 11,9 & 21,5 \\
Menta spearmint & 16,2 & 43,2 & 31,9 & 34,5 & - & 62,4 \\
Otras mentas & - & - & - & 71,2 & 51,7 & 86,8 \\
Palma rosa & - & 54 & 38,6 & - & 283 & - \\
Palo rosa & 8,5 & 22,6 & 5,7 & 192,6 & 221 & 238,6 \\
Palo santo & - & 179,4 & 170,8 & - & - & - \\
Vetiver & 22,2 & 63,1 & 28 & 271,2 & 397,6 & 17,9 \\
\hline
\end{tabular}

Fuente: Datos extraídos de AliceWeb, 2016.

\section{Resultados y Discusión}

\section{Valores en dólares acumulados}

Los valores totales acumulados de las exportaciones en dólares se organizaron de mayor a menor valor para cada uno de los 16 aceites esenciales seleccionados (Figura 2). Se destacó el aceite de palo rosa, lavanda y vetiver, en las tres primeras posiciones.
Adicionalmente, se resaltó que de los 26 estados brasileños, solo 15 de ellos entraron en el registro de estados que exportan aceites esenciales. San Pablo, Amazonas, Minas Gerais, Paraná y Rio Grande del Norte, ocupan las primeras cinco posiciones de mayores exportadores en kilogramos y dólares en los valores acumulados durante 2000 hasta 2015 (Tabla 3).

San Pablo se destacó en la exportación de los 16 aceites esenciales seleccionados, mientras el 


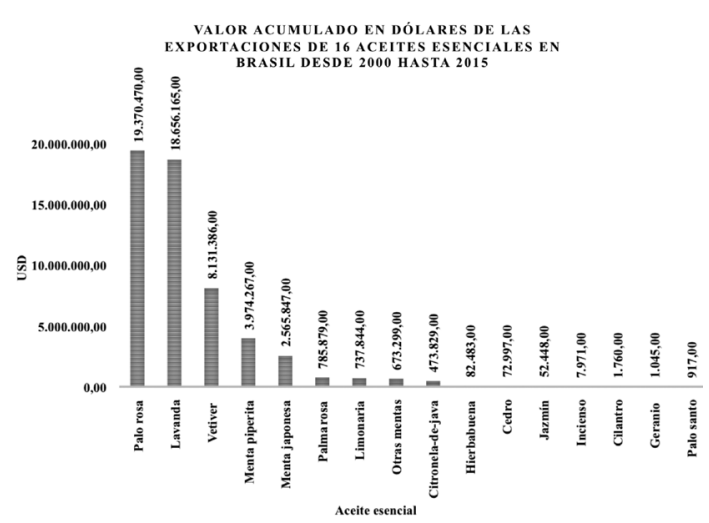

Figura 2. Valor acumulado en dólares (USD) de las exportaciones de aceites esenciales en el Brasil entre el período de 2000 hasta 2015.

estado del Amazonas se posiciono en la exportación de aceite esencial de palo rosa y lavanda.

A continuación, en la Tabla 3, se observan los dos primeros productos con mayores valores en dólares y kilogramos para los 15 estados brasileños registrados.

Amazonas sobresale por el mayor valor en exportaciones en dólares de aceite esencial de palo rosa (USD 15.583.512). Entre tanto, San Pablo presentó el más alto valor en kilogramos con $1.374 .349 \mathrm{~kg}$ de aceite esencial de lavanda.

Del mismo modo, Minas Gerais tiene registro más alto de exportaciones de lavanda (USD 2.741.700) y vetiver (USD 1.302.753). Asimismo, Paraná se destacó con el aceite de menta japonesa (USD 1.317.325) y lavanda (USD 1.030.578) y el estado Rio Grande do Norte se destacó en la exportación de vetiver (USD 2.494.920) y palma rosa (USD 18.144) (Tabla 3).

El estado brasileño de Pará, exportó aceite esencial de lavanda, menta piperita, palo rosa y vetiver; Rio Grande do Sul envió AE de citronelade-java, otras mentas, limonaria y lavanda; Rio de Janeiro exportó lavanda, citronela-de-java y, en menor proporción, incienso, cedro, hierbabuena, jazmín, otras mentas, limonaria, menta japonesa, vetiver y menta piperita; Ceará facturó las denominadas otras mentas y lavanda; Santa Catarina se destacó con otras mentas, lavanda, menta piperita y cilantro; Mato Grosso do Sul se destacó en la exportación de incienso, lavanda, menta japonesa, palma rosa y limonaria.; el estado de Espírito Santo con menta japonesa, otras mentas, limonaria y lavanda. Por último, Goiás, Bahía y Rondonia apenas tuvieron presencia en la exportación de lavanda.
Tabla 3. Valores acumulados de los 15 estados brasileños con los dos aceites esenciales que ocuparon las primeras posiciones en exportaciones en dólares y kilogramos de producto desde 2000 hasta 2015.

\begin{tabular}{|c|c|c|c|}
\hline Estado & Especie & USD & $\mathrm{kg}$ \\
\hline \multirow{2}{*}{ San Pablo } & Lavanda & 11.271 .846 & 1.374 .349 \\
\hline & Menta piperita & 3.841 .597 & 134.409 \\
\hline \multirow{2}{*}{ Amazonas } & Palo rosa & 15.583 .512 & 238.852 \\
\hline & Lavanda & 3.364 .618 & 95.701 \\
\hline \multirow{2}{*}{ Minas Gerais } & Lavanda & 2.741 .700 & 355.209 \\
\hline & Vetiver & 1.302 .753 & 89.462 \\
\hline \multirow{2}{*}{ Paraná } & Menta japonesa & 1.317 .325 & 96.660 \\
\hline & Lavanda & 1.030 .578 & 76.629 \\
\hline \multirow{2}{*}{$\begin{array}{l}\text { Rio Grande del } \\
\text { Norte }\end{array}$} & Vetiver & 2.494 .920 & 23.750 \\
\hline & Palma rosa & 18.144 & 566 \\
\hline \multirow{2}{*}{ Pará } & Lavanda & 122.101 & 1.018 \\
\hline & Menta piperita & 121.868 & 1.010 \\
\hline \multirow{2}{*}{$\begin{array}{l}\text { Rio Grande } \\
\text { do Sul }\end{array}$} & Citronela-de-java & 259.675 & 13.719 \\
\hline & Otras mentas & 5.906 & 364 \\
\hline \multirow{2}{*}{ Rio de Janeiro } & Lavanda & 107.750 & 3.505 \\
\hline & Citronela-de-java & 44.186 & 2.109 \\
\hline \multirow{2}{*}{ Ceará } & Otras mentas & 55.253 & 2.370 \\
\hline & Lavanda & 10 & $\overline{1}$ \\
\hline \multirow{2}{*}{ Santa Catarina } & Otras mentas & 12.993 & 816 \\
\hline & Lavanda & 11.032 & 616 \\
\hline \multirow{2}{*}{$\begin{array}{l}\text { Mato Grosso } \\
\text { do Sul }\end{array}$} & Incienso & 468 & 18 \\
\hline & Lavanda & 65 & 2 \\
\hline \multirow{2}{*}{ Espíritu Santo } & Menta japonesa & 437 & 38 \\
\hline & Otras mentas & 17 & 5 \\
\hline Goiás & Lavanda & 4.250 & 100 \\
\hline Bahía & Lavanda & 1.800 & 200 \\
\hline Rondonia & Lavanda & 135 & 12 \\
\hline
\end{tabular}

Fuente: Datos del sitio web AliceWeb, 2016.

\section{Selección y comportamiento de los diez aceites esenciales más comercializados}

Los diez AE con mayores valores de exportación en dólares (del más alto al más bajo) fueron: palo rosa, lavanda, menta piperita, vetiver, menta japonesa, palma rosa, limonaria, otras mentas, citronela-de-java y hierbabuena (Figura 2). 
En la Figura 3 puede observarse el comportamiento de las exportaciones en relación a los kilogramos y dólares comercializados. Se observó que las especies palo rosa, menta piperita, vetiver y otras mentas presentaron claras oscilaciones en sus exportaciones. Lo anterior probablemente es resultado de dos factores: 1) Los productores de estas especies son como mencionó Bandoni (2000), productores pequeños con menos de 10 ha, que no están asociados a cooperativas y donde el cultivo

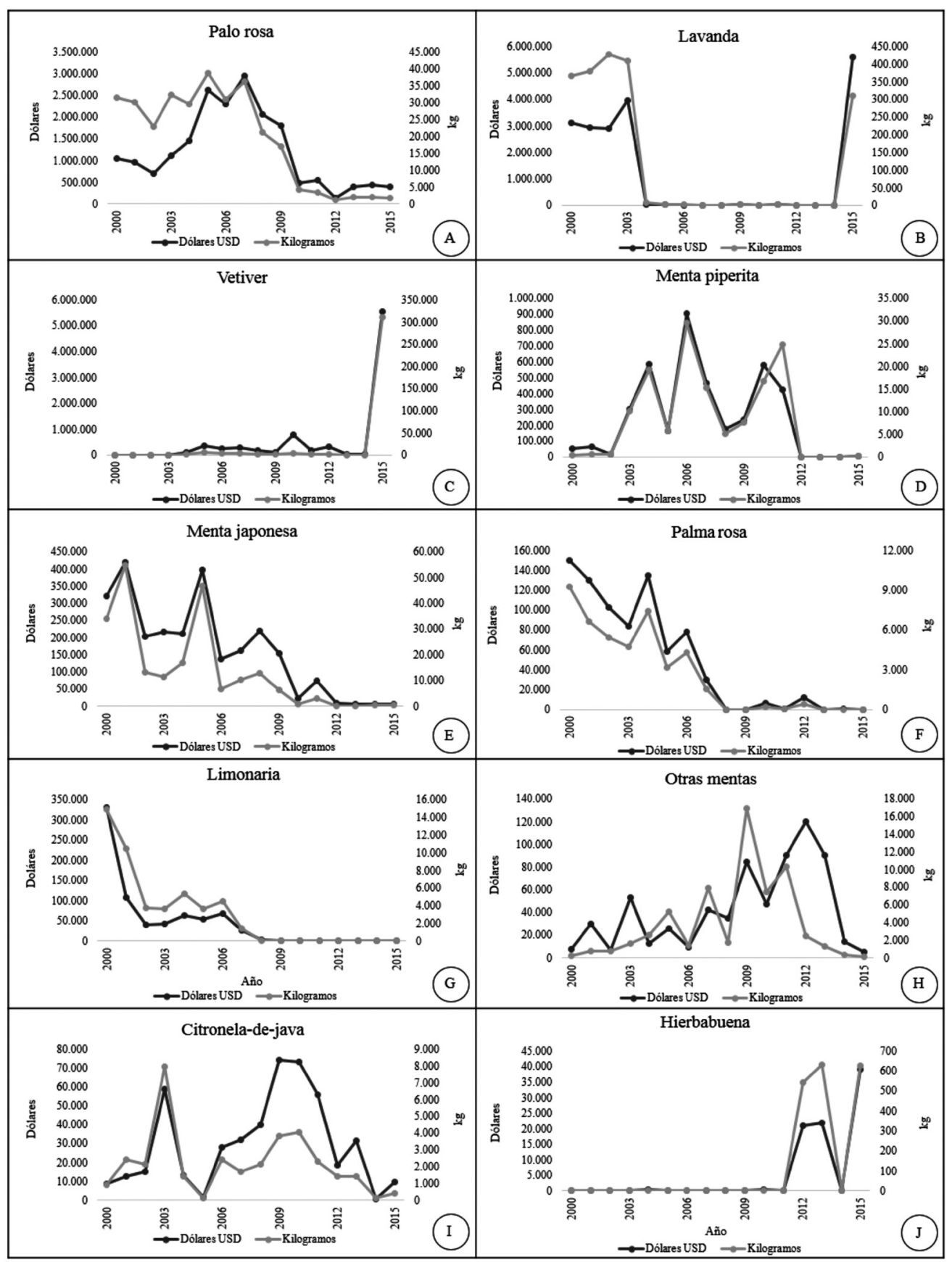

Figura 3. Exportaciones en dólares y kilogramos desde 2000 hasta 2015, de los 10 primeros aceites esenciales que presentaron los valores más altos acumulados en la suma total de las exportaciones. Fuente: AliceWeb, 2016. 
de estas especies son una alternativa paralela a otro cultivo tradicional dentro de sus propiedades, y 2) La presencia de Brokers que movilizan grandes cantidades de mercancía y funcionan como centralizadores, y son ellos los que regulan los precios y determinan los períodos de venta.

El aceite esencial de palo rosa (Aniba roseadora), presentó sus más altos valores de exportación en dólares entre los años 2000 hasta 2009 (variando entre 16.000 a $38.000 \mathrm{~kg} \mathrm{año}^{-1}$ ) (Figura 3A).

El aceite esencial de lavanda presento sus picos de exportación entre los años de 2000 hasta 2003 (366.936 a $\left.426.303 \mathrm{~kg} \mathrm{año}^{-1}\right)$ y en 2015 (310.838 kg año ${ }^{-1}$ ) (Figura 3B). El aceite de vetiver tuvo la exportación más alta en 2015 con $309.902 \mathrm{~kg} \mathrm{año}^{-1}$, los otros años no sobrepasó los $5.210 \mathrm{~kg} \mathrm{año}^{-1}$ (2005) (Figura 3C).

Las exportaciones de menta piperita se caracterizaron por su constante irregularidad y diversos picos en el transcurrir de estos quince años (Figura 3D). Su mayor valor fue en $2006(29.581 \mathrm{~kg}$ año ${ }^{-1}$ ), seguido del $2011\left(24.744 \mathrm{~kg}\right.$ año $\left.{ }^{-1}\right)$.

La menta japonesa (Figura 3E) presentó heterogeneidad en sus exportaciones, siendo que ha disminuido al transcurrir del tiempo; la mayor exportación fue en 2001 (54.675 $\mathrm{kg}$ año ${ }^{-1}$ ), luego en 2005 (46.780 kg año $\left.{ }^{-1}\right)$, y su última mayor exportación en 2008 (12.650 $\mathrm{kg}$ año $\left.^{-1}\right)$. Un escenario similar ocurrió con palma rosa (Figura 3F), donde en 2000 obtuvo $9.255 \mathrm{~kg}_{\text {año }}{ }^{-1}$, seguido del 2004 (7.399 kg año $\left.{ }^{-1}\right)$, y $2006(4.279 \mathrm{~kg}$ año-1), con valores decrecientes en las exportaciones.

La limonaria (Figura 3G) presentó una disminución de sus exportaciones en el intervalo de tiempo analizado, donde sus picos en kilogramos fueron en los años 2000, 2004 y 2006 (14.894; $5.330 ; 4.531 \mathrm{~kg} \mathrm{año}^{-1}$, respectivamente).

El aceite esencial clasificado como "otras mentas" presentó un comportamiento irregular durante este intervalo de tiempo analizado (Figura 3H), no obstante la tendencia general mostró que hasta el 2009 hubo crecimiento. Durante ese horizonte temporal la producción se incrementó alcanzando su más alto valor en kilogramos en el 2009 con $16.889 \mathrm{~kg} \mathrm{año}^{-1}$.

Citronela-de-java es otro aceite esencial que no presentó alguna homogeneidad en sus exportaciones, sus más altos valores se encontraron en 2003 (7.977 $\mathrm{kg}$ año $\left.{ }^{-1}\right), 2009$ (3.847 $\left.\mathrm{kg} \mathrm{año}{ }^{-1}\right)$ y 2010 (4.042 $\mathrm{kg}$ año $\left.^{-1}\right)$ (Figura 3I).
El aceite esencial de hierbabuena mostró participaciones esporádicas en el transcurso del tiempo (Figura 3J), en el año 2012 hubo una exportación de $540 \mathrm{~kg}$ año ${ }^{-1}$, para el $2013 \mathrm{de}$ $630 \mathrm{~kg}$ año ${ }^{-1}$ y 2015 con $626 \mathrm{~kg}$ año ${ }^{-1}$.

Por otro lado, aunque Brasil no tiene una producción establecida de lavanda, reexporta este producto, el que ocupa la segunda posición en sus exportaciones en comparación con los 16 aceites esenciales seleccionados en el intervalo de tiempo analizado.

Es probable que estas oscilaciones en la exportación de aceites esenciales de vetiver, menta piperita, otras mentas, citronela-de-java (Figura 3C, 3D, 3H, 3I, respectivamente) fueran el resultado de la interacción de varios requisitos ambientales, técnicas de cultivo, fertilización y prácticas agrícolas, que escasearon en planificación previa para que el productor pudiera dar continuidad en el suministro de aceite esencial y donde, es probable, existieron deficiencias.

\section{Valor del kilogramo}

Se observa en la Tabla 2 , el valor del kilogramo en 2000, y desde 2011 hasta 2015 para cada uno de los 16 aceites esenciales seleccionados, destacando el AE en 2013 de vetiver 271,2 USD kg-1 y palo de rosa 196,6 USD kg-1. Para el 2015 fue el AE vetiver por $397,6 \mathrm{USD} \mathrm{kg}^{-1}$ y palma rosa con 283,0 USD kg-1.

Es primordial mencionar que este estudio exploratorio que entrega una aproximación general que analizó solamente 16 aceites esenciales, donde se determinó que los estándares de exportaciones no ocurren de manera uniforme y responden a diferentes normas de la industria como lo indican (Amurgo-Pacheco y Pierola, 2008). Además, se debe tener en cuenta que las especies involucran diferentes hábitos de crecimiento y distintas formas de gestión. Del mismo modo, la Dirección de Inteligencia Comercial e Inversiones (2011) identifica que no existe un patrón en la frecuencia de compra de los aceites esenciales.

Los aceites esenciales son productos agroindustriales no tradicionales, lo que significa un mercado limitado y con restricciones en el acceso a fuentes de información y fuentes de consumo.

Se resalta que una de las dificultades que surgió en la realización del ejercicio fue la ausencia de entidades reconocidas para extraer 
la información, conocer los lugares de trabajo generados por esta industria e incluso saber detalles de las especies usadas para la obtención de los AE.

Fueron excluidos los denominados "otros aceites esenciales" presentes en la base de dados del sistema AliceWeb, donde quizás se eliminaron datos e informaciones relacionadas con especies relevantes exportadas; y aunque pueden tener volúmenes pequeños, presentan un altísimo potencial por ser distintivos del país.

Se observa que el estado de San Pablo y sus altos valores de exportación en dólares y kilogramos de aceites esenciales, puede considerarse como el gran Broker o intermediario en Brasil, donde se concentra la venta de los 16 aceites esenciales seleccionados, posicionándolo como el estado centralizador de las exportaciones en Brasil.

Las estrategias de mercado son muy importantes cuando se considera alcanzar los objetivos industriales: disponibilidad con alta calidad y estabilidad en los precios del producto. No obstante, el mercado de exportación de estos 16 aceites esenciales enfrenta estos dos objetivos para mejorar, con base a las fluctuaciones de exportaciones que los productos presentaron en el transcurso de este intervalo de tiempo. Probablemente, el resultado de las diferencias encontradas desde que se inicia la fase de cultivo de la especie, donde interfieren factores como la forma de propagación de la especie, la colecta y extracción del aceite esencial y diversos rendimientos; así como las diversas formas de comercialización que comienza desde los pequeños productores hasta los grandes intermediarios Brokers que actúan en la venta, de igual forma, las oscilaciones de los compradores para con los aceites esenciales.

\section{Conclusiones}

La heterogeneidad en el comportamiento de las exportaciones de los aceites esenciales en Brasil impide el establecimiento de un patrón de comportamiento de los productos seleccionados para este intervalo de tiempo.

\section{Agradecimientos}

El primer autor agradece el apoyo financiero proporcionado por el Consejo Nacional de Desarrollo Científico y Tecnológico, CNPq, Brasil, Beca 142052 / 2014-0.

\section{Literatura Citada}

Alice Web

2016 [En línea]: Brasilia: MDIC - Sistema de Análisis de Informaciones de Comercio Exterior Vía Internet del Ministerio del Desarrollo, Industria y Comercio Exterior. AliceWeb. Disponible en: http://aliceweb.desenvolvimento. gov.br. Acceso en: Feb/2016.

Amurgo-Pacheco, A.; Pierola, M.D. 2008. Patterns of Export Diversification in Developing Countries: Intensive and Extensive Margins. Policy Research Working Paper 4473. The World Bank. Washington, DC. USA, 32 p.

Bandoni, A.

2000. Los recursos vegetales aromáticos en Latinoamérica. $\mathrm{Su}$ aprovechamiento industrial para la producción de aromas y sabores. Editorial Universidad Nacional de La Plata-Cooperación Iberoamericana CYTED. Buenos Aires, Argentina, 410 p.

Biocomercio Sostenible

2003. Estudio del mercado colombiano de aceites esenciales. Instituto de Investigación de Recursos Biológicos Alexander von Humboldt, Bogotá, Colombia, 109 p.

Dirección de Inteligencia Comercial e Inversiones. Pro Ecuador. 2011. Perfil de aceites esenciales en Estados Unidos. Ministerio de Relaciones Exteriores, Comercio y Integración. Quito, Ecuador, $52 \mathrm{p}$.
Erkekdjian, M.

2002. Análisis de la tendencia del mercado internacional de aceites esenciales. Informe mensual de la Fundación Export-Ar. Bs. Ar., Argentina, 24 p.

Fretes, F.; Martínez, M.

2011. Aceites esenciales: Análisis de la cadena de valor. Agencia del Gobierno de los Estados Unidos para el Desarrollo Internacional (USAID/Paraguay). 48 p. Disponible en: https://www.usaid.gov/sites/default/files/ documents/1862/aceites_esenciales.pdf. Acceso en: Feb/2016.

Figueiredo, A.C.; Barroso, J.G.; Pedro, L.G.; Scheffer, J.J.

2008. Factors affecting secondary metabolite production in plants: Volatile components and essential oils. Flavour and Fragrance Journal, 23: 213-226.

Gueudar, F.D.

2013. Production et marchés des huiles essentielles de lavandes et lavandins. Plantes à parfum, aromatiques et médicinales. [On line]. France Agri Mer. (1): 8-12. Disponible en: http:// www.franceagrimer.fr/content/download/26324/221437/ file/Synth\%C3\%A8se\%20PPAM\%20n\%C2\%B01\%20 -\%20Juin\%202013.pdf. Acceso en: Nov/2015.

Ideaconsultora

2011. Consultoría: Potencial de producción y exportación del sector agroindustrial hortofrutícola. Informe Final. 
Oficina de Estudios y Políticas Agrarias (Odepa). Santiago, Chile, $582 \mathrm{p}$.

Khodjet-El-Khil, M.

2015. Étude de marché: Consommation des huiles essentielles en Guyane Française. Direction de l'Alimentation, de l'Agriculture et de la Forêt (DAAF). Cayena, Guayana Francesa, 36 p.

Lange, D.

1998. Europe's medicinal and aromatics plants. Their use, trade and conservation. Traffic international Ed. Cambridge, $\mathrm{UK}, 77 \mathrm{p}$

Laszlo, F.F. (s.f.)

Naturais x Sintéticos. [On line]: Laszlo Aromaterapia Ltda. Disponible en: http://laszlo.ind.br/default. asp?pagina=naturais_sinteticos. Acceso en: Feb/ 2016.

SADC Trade Development Programme

2005. Trade Information Brief. Essential oils. Trade and Industrial Policy Strategies (TIPS) and Australian Agency for International Development (AUSAID). Pretoria, Sudáfrica, 48 p.
Silva, R.C.; Fátima, L.; Costa, L.G.

2012. Ferramentas de TI no ensino e aprendizagem da sistemática de comércio exterior. In: Congresso Norte Nordeste de Pesquisa e Inovação CONNEPI VII, 19-21 de octubre de 2012. Palmas - Tocantins - Brasil, Resume, 6 p.

United Nations Conference on Trade and Development. UNCTAD. 2005. Market brief in the European Union for select natural ingredients derived from species. Lippia alba Pronto alivio, erva cidreira, juanilama, melissa. Ginebra, Suiza, $39 \mathrm{p}$.

Verlet $\mathrm{N}$.

1992. The world herbs and essential oils economy - analysis of the medium term development. Acta Horticulturae, 306: 474-481.

Weiss, E.A.

1997. Essential oil crops. Ed. CAB International. Wallingford, $\mathrm{UK}, 600 \mathrm{p}$. 